\title{
Intercooperação e fusão de redes empresariais: proposição de framework para análise sob a perspectiva da aprendizagem
}

\section{Intercooperation and merger of business networks: proposition of a framework based on learning approach}

DOUGLAS WEGNER

Universidade do Vale do Rio dos Sinos - UNISINOS HERON SERGIO MOREIRA BEGNIS Universidade de Santa Cruz do Sul - UNISC

ANELISE REBELATO MOZZATO

Universidade de Passo Fundo (UPF)

\section{RESUMO}

Este ensaio aborda a estratégia de intercooperação e fusão de redes empresariais sob a perspectiva sociológica da aprendizagem da rede. A cooperação entre redes consiste em uma estratégia para acelerar o crescimento e buscar a consolidação das empresas integrantes. No entanto, sua execução depende de um processo de aprendizagem da rede, em que as participantes aprendem enquanto grupo sobre a necessidade de cooperar com outras redes. Com base no processo de aprendizagem da rede, o ensaio apresenta e discute um framework para analisar a intercooperação e a fusão de redes empresariais, salientando os episódios de aprendizagem que ocorrem tanto na implementação como na consolidação da estratégia. São apresentadas premissas e três proposições que permitem analisar o contexto, o conteúdo e o processo da aprendizagem da rede. O framework contribui para a compreensão do fenômeno da aprendizagem da rede e como esta interfere na estratégia de intercooperação e fusão de redes. Palavras-chave: Relações interorganizacionais. Intercooperação. Fusão de redes. Aprendizagem da rede. Perspectiva sociológica da aprendizagem. 


\section{Abstract}

The paper addresses the strategy of intercooperation and merger of business networks, based on the sociological perspective of network learning. Cooperation among business networks consists of a strategy to accelerate networks' growth and consolidation. However, the implementation of this strategy depends on a network learning process, in which the firms learn as a group on the need to cooperate with other business networks. This paper presents a framework for analyzing the intercooperation and merger of business networks. It is based on the network learning process and the identification of learning episodes that occur in the strategy implementation and consolidation. We present assumptions and three propositions to analyze the context, content and process of network learning. The framework contributes to the understanding of network learning and how this phenomenon directs the network intercooperation and mergers strategies. Key-words: Interorganizational relations. Intercooperation. Merger. Nertwork learning. Sociological perspective.

\section{INTRODUÇÃo}

Como resposta à pressão do ambiente competitivo muitas organizações têm optado pelo estabelecimento de relações interorganizacionais de diversos formatos e características (TODEVA, 2006), por meio das quais essas organizações conseguem acessar ou desenvolver recursos críticos à competição, obter melhor posição no mercado ou gerar inovações de forma colaborativa (HUIZINGH, 2011; LICHTENTALER, 2011). Estabelecer relações interorganizacionais tem sido uma prática comum entre as grandes empresas, como se observa em setores tradicionais, a exemplo do transporte aéreo (LAZZARINI, 2007) e a indústria automotiva (SCHULZE; BROJERDI; KROGH, 2014). Entre as pequenas empresas as relações interorganizacionais configuram-se principalmente na forma de redes de cooperação (KILDUFF; TSAI, 2003; GRONUM; VERREYNNE; KASTELLE, 2012).

Tal é a importância que as redes interorganizacionais representam para as empresas que a competição não está mais confinada ao nível das firmas, mas caracteriza-se como um fenômeno no nível 
das redes (GOMES-CASSERES, 1994; LAZZARINI, 2007). Nesse contexto, fortalecer a rede é indispensável para competir. Para isso cada rede precisa crescer em número e diversidade de participantes, bem como desenvolver recursos e competências que a tornem mais atrativa do que as concorrentes, sob pena de perder seus membros ou ser incapaz de atrair novos partícipes. O número de membros gera efeitos de escala, favorecendo a negociação com fornecedores e clientes, bem como o estabelecimento de barreiras à competição. A diversidade de composição de componentes, por sua vez, pode favorecer a aprendizagem e acelerar a inovação (BALESTRIN; VERSCHOORE 2010) e o desenvolvimento de competências.

Os benefícios proporcionados pela cooperação às pequenas empresas estão refletidos no grande número de redes criadas no Brasil nos últimos anos. Conforme o Serviço Brasileiro de Apoio às Micro e Pequenas Empresas (SEBRAE, 2012) foram criados mais de 800 redes de empresas no país em uma década. Embora a consolidação seja um objetivo de todas essas redes, estudos têm revelado que muitas redes de pequenas empresas têm dificuldades para se manter em atividade devido ao pequeno número de membros (TOIGO; ALBA, 2010; WEGNER; PADULA, 2012; SEBRAE, 2012) e à incapacidade de constituir uma estrutura profissional de gestão da rede (WEGNER; ALIEVI; BEGNIS, 2015).

Uma possível estratégia de consolidação de redes empresariais é a intercooperação ou a fusão delas, visando ganhos de escala e a potencialização de resultados. Essas estratégias proporcionam sinergias entre as redes, permitindo melhores negociações com fornecedores, ampliação do portfólio de serviços ofertados aos participantes e melhores condições de sustentar uma estrutura de gestão com profissionais contratados (GRO $\beta$, 2003; WEGNER; PADULA, 2010; WEGNER; PADULA, 2011). No entanto processos de colaboração mútua e união envolvem significativos desafios, dadas as características mercadológicas, estratégicas e culturais das redes envolvidas, gerando questões ainda não respondidas pela teoria organizacional. Embora a literatura seja proeminente em estudos que analisam os motivos para a cooperação e os resultados alcançados (BRASS; GALASKIEWICZ; GREVE, 2004; YSA; SIERRA; ESTEVE, 
2014), são raros os estudos que buscam compreender como ocorre a colaboração entre redes (SOARES; WEGNER; DOLCI, 2016).

Mais do que isso, até o momento não foram realizados estudos para compreender como a aprendizagem da rede está relacionada à decisão de iniciar um processo de intercooperação e fusão entre redes. Neste ensaio parte-se do pressuposto que o processo de aprendizagem da rede, denominado de network learning por Knight (2002), Knight e Pye (2005) e White (2008), pode facilitar as estratégias de intercooperação e fusão entre redes, uma vez que estas envolvem o processo de aprendizagem para que sejam conduzidas e implementadas com sucesso. Com base nessa contextualização, este ensaio teórico apresenta uma proposta de framework para aprofundar o entendimento sobre o processo de intercooperação e fusão de redes interorganizacionais a partir da aprendizagem da rede, sob a perspectiva sociológica da aprendizagem.

O texto está organizado em quatro seções, além desta introdução. Na segunda apresentam-se as características das redes de pequenas empresas e as estratégias de intercooperação e fusão. $\mathrm{Na}$ terceira expõe-se a abordagem da aprendizagem da rede na perspectiva sociológica, a qual é utilizada como abordagem teórica de base para a proposição do framezwork de pesquisa, que está na quarta parte. As considerações finais do ensaio são apresentadas na quinta seção, incluindo direções para estudos futuros.

\section{ESTRATÉGIAS DE INTERCOOPERAÇÃO DE REDES}

As redes interorganizacionais foco deste estudo são caracterizadas como grupos compostos por empresas que possuem objetivos comuns e relações formais, com prazo ilimitado de existência e manutenção da individualidade legal dos participantes (KILDUFF; TSAI, 2003). Ao contrário de redes verticais ou cadeias de suprimento (LAMBERT; COOPER; PAGH, 1998; GULATI, 1998) em redes horizontais formadas por pequenas empresas geralmente inexiste a figura da organização focal que coordena as demais. Essas redes caracterizam-se por várias etapas de constituição e desenvolvimento (CHILD, 2001) com estruturas de natureza dinâmica e que se modificam ao longo do tempo (EBERS; GRANDORI, 1999; WEGNER; MISOCSKY, 2010). 
A adoção de estratégias para ampliar o número de participantes e o desenvolvimento de novas competências e recursos é fundamental para a sobrevivência das redes, seja para manter o interesse dos participantes como também para atrair novos membros. Diversas estratégias podem ser adotadas por redes empresariais com vistas a gerar crescimento $(\mathrm{GRO} \beta$, 2003). Duas são denominadas estratégias básicas que objetivam o crescimento da rede por meio da penetração de mercado e do aumento de eficiência. A primeira refere-se a captar novos membros para ampliar a rede e a segunda busca otimizar os resultados com os membros atuais, procurando aumentar a fidelidade deles com a rede.

Embora as duas estratégias básicas de crescimento possam gerar resultados de curto prazo, Gro $\beta$ (2003) acredita que elas não são solução de longo prazo para as necessidades das redes. Como complemento o autor apresenta estratégias com foco externo à rede: diversificação de segmentos, ampliando o escopo da rede; horizontalização, por meio de parcerias e fusões com outras redes; verticalização, agregando outros elos da cadeia; e internacionalização, partindo para a expansão em outros países. A estratégia de horizontalização, executada por parceiras, intercooperações e fusões com redes já existentes, pode gerar o crescimento com menores riscos porque internaliza a experiência dos novos parceiros. A adoção dessa estratégia de crescimento é relatada tanto na literatura internacional (WELLENBECK, 2001) quanto na nacional (WEGNER; A LIEVI; BEGNIS, 2015).

A intercooperação é originalmente tratada como um princípio básico do cooperativismo e consiste em promover a aproximação entre cooperativas para a busca de benefícios coletivos (ROSSI, 2005). Embora redes interorganizacionais tenham características distintas de cooperativas, entende-se que o conceito de intercooperação também se aplica a elas tendo em vista as possibilidades de ampliação dos ganhos coletivos. De modo geral, a intercooperação consiste na formação de alianças entre organizações de caráter colaborativo. Trata-se de uma aliança no nível das redes já existentes, podendo ser denominada como uma relação inter-redes. A Figura 1 (esquerda) apresenta o processo de intercooperação de redes em que empresas individuais formam redes que, por sua vez, cooperam 
entre si. Nesse modelo cada rede mantém sua individualidade (Rede A, Rede B, Rede C), mas ocorrem ações conjuntas que buscam beneficiar as empresas participantes das três redes. Não obstante haja colaboração no nível das redes, é possível que ocorram relações de competição entre os membros individuais, por ocuparem posições próximas no mercado.

Uma etapa seguinte da intercooperação pode ser a fusão entre redes. Nesse caso, ocorrem mais do que ações conjuntas entre elas, na medida em que passam a formar uma única rede, deixando de existir individualmente. A Figura 1 (direita) apresenta o processo de fusão de redes, em que as redes originais (Rede A, B e C) deixam de existir e forma-se a Rede D.

\section{Figura 1 - Intercooperação e fusão de redes de empresas}

INTERCOOPERAÇÃO DE REDES EMPRESARIAIS

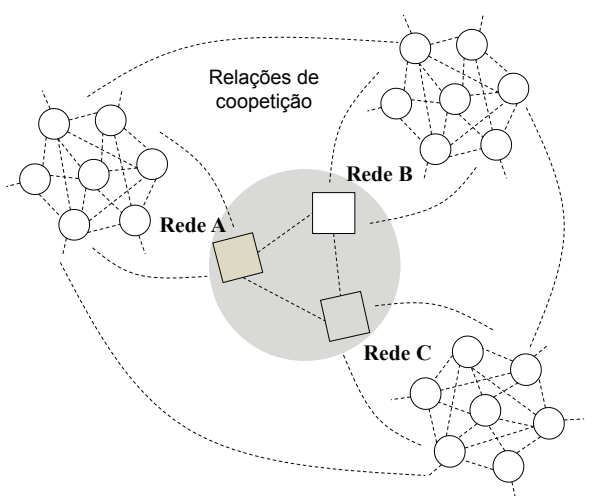

Fonte: Elaborada pelos autores

\section{REDES EMPRESARIAIS}

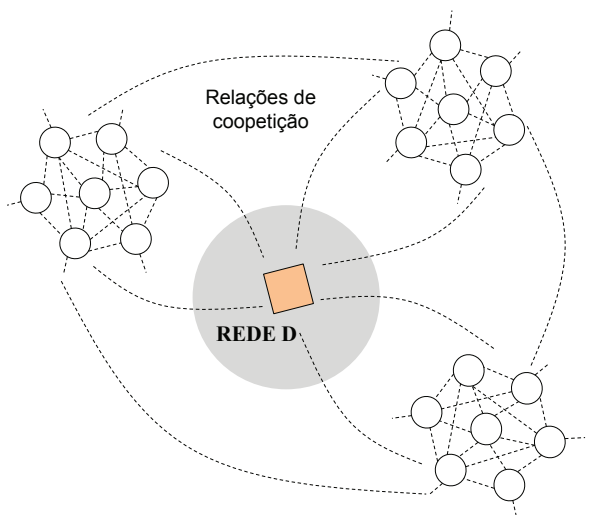

Em estratégias de intercooperação e fusão de redes o processo da aprendizagem ocorre nas fronteiras organizacionais, constituindo-se como importante para a sua efetiva implementação, condução e consolidação. Na implementação as empresas participantes aprendem enquanto grupo sobre a necessidade de buscar a aproximação com outras redes, por meio da geração de episódios de aprendizagem, enquanto na condução os episódios de aprendizagem são corriqueiros por meio da interação entre as redes. Já na consolidação 
a aprendizagem da rede acontece em razão da certeza das empresas quanto à importância em participar da intercooperação. À medida que a intercooperação ocorre de maneira bem-sucedida podem advir reforços de aprendizagem que levam à fusão entre as redes. Portanto apresenta-se na sequência a perspectiva sociológica da aprendizagem nas organizações com foco nos episódios de aprendizagem, que se dão por meio das interações dos atores.

\section{Perspectiva Sociológica da APREndizagem ORGANIZACIONAL}

O tema da aprendizagem organizacional (AO) é abordado por meio de diferentes concepções na literatura de gestão, como a perspectiva psicológica (MAIER; PRANGE; ROSENTIEL, 2001), econômica (BOERNER, C.; MACHER, J.; TEECE, 2001), antropológica (CZARNIAWSKA, 2001) e sociológica (GHERARDI; NICOLINI; ODELLA, 1998; GHERARDI; NICOLINI, 2001). A perspectiva psicológica trata a AO como uma analogia da aprendizagem individual ou então a aprendizagem individual é considerada como base para a organizacional (MAIER; PRANGE; ROSENSTIEL, 2001). Na abordagem econômica a aprendizagem é analisada pela perspectiva do incremento no desempenho econômico da organização. Por sua vez, na abordagem antropológica as organizações são entendidas como culturas e como processos, e a aprendizagem ocorre por meio de mudanças nos artefatos culturais da organização (CZARNIAWSKA, 2001).

O campo da gestão organizacional também tem utilizado a abordagem sociológica, que destaca a noção de participação e reflexividade nas práticas cotidianas (GHERARDI; NICOLINI; ODELLA, 1998; GHERARDI, 2012; BERENDS; ANTONACOPOULOU, 2014). A ideia de participação enfatiza que a aprendizagem não acontece somente na mente dos indivíduos, mas sim a partir da atuação desses em atividades sociais.

A reflexividade está diretamente ligada à participação, porque ocorre quando o fluxo de experiência é interrompido e o sujeito reflete sobre o conhecimento. Refere-se às maneiras de ver o ato passado e reflete os modos existentes de ver (GHERARDI; NICOLINI, 2001). Logo, participação denota a existência de interação 
e compartilhamento de normas, valores, identidades e significados por um grupo de indivíduos, gerando aprendizagem. Tanto a participação como a reflexividade facilitam a aprendizagem, a qual se dá nas práticas cotidianas que ocorrem no todo relacional (GHERARDI; NICOLINI; ODELLA, 1998; LAVE; WENGER, 1991; GHERARDI; NICOLINI, 2001; NICOLINI; GHERARDI; YANOW, 2003; MASON; LEEK, 2008; CORRADI; GHERARDI; VERZELLONI, 2010; BERENDS; ANTON ACOPOULOU, 2014; GHERARDI, 2015).

$\mathrm{Na}$ abordagem sociológica a aprendizagem e o conhecimento não são vistos apenas na linha da cognição, mas como construções sociais nas ações diárias. O conceito de práticas é central, afirmando que são essas que possibilitam a aprendizagem, independentemente do nível - individual, grupal ou organizacional e mesmo interorganizacional (GHERARDI; 2009a, 2009b, 2012, 2015).

Como referem Mason e Leek (2008), as pessoas aprendem umas com os outras e tal processo pode desenvolver o que denominam de "comunidade de práticas interempresa". Para Gherardi (2009b, 2012), as práticas oportunizam o processo da aprendizagem e também a organização de um grupo. Tais práticas constituem-se em ações que acontecem no fluxo cotidiano das atividades, tanto em espaços sociais estruturados (formais) como não estruturados (informais/casuais), desencadeando a aprendizagem (STYHRE; JOSEPHSON; KNAUSEDER, 2006; CORRADI; GHERARDI; VERZELLONI, 2010; JANOWICZ-PANJAITAN; NOORDERHAVEN, 2009; SANDBERG; TSOUKAS, 2011). “As situações sociais casuais são tão importantes quanto as experiências de aprendizagem formal" (GHERARDI, 2006, p.46).

Tanto em situações formais como informais de aprendizagem a participação se dá por meio da interação entre indivíduos em diferentes espaços sociais, os quais envolvem comunicação, diálogo e reflexividade (LARSSON et al., 1998; MACDONALD; CROSSAN, 2010). É por meio do diálogo que são desenvolvidos entendimentos comuns que facilitam a aprendizagem interorganizacional (AIO) (MACDONALD; CROSSAN, 2010),

Nesse contexto a aprendizagem se dá no fluxo de atividades que acontecem no cotidiano, ficando evidenciada a aprendizagem 
como processo (LAVE; WENGER, 1991; EASTERBY-SMITH, 1997; GHERARDI; NICOLINI, 2001) em seus diferentes níveis: individual, grupal, organizacional e interorganizacional. Em todo o processo há pessoas envolvidas, motivo pelo qual emoções, aspectos positivos, poder e dominação interferem no processo de aprendizagem (GHERARDI; 2006, 2009a, 2009b, 2012; MACDONALD; CROSSAN, 2010; CHIVA; HABIB, 2015).

A aprendizagem é compreendida pelo emergir de relações e interações das pessoas com os elementos sociais e materiais de contextos particulares. Hibbert et al. (2010) concordam com Antonacopoulou (2006) e Antonacopoulou e Chiva (2007) quanto ao fato de que, para se compreender a aprendizagem necessariamente devem ser considerados os aspectos individuais, relacionais e sociais dos processos situados de aprendizagem (Sim, está correto). Fatores sociais e relacionais são básicos para que ocorra a aprendizagem coletiva; a ampliação das conexões sociais aumenta as possibilidades de aprendizagem (HIBBERT et al., 2010). De maneira semelhante as empresas em redes compostas por parceiros com experiências heterogêneas estarão em melhor posição para se beneficiar dessas experiências do que empresas em redes compostas de parceiros com experiências homogêneas (CORSARO; CANTÙ; TUNISINI, 2012). Por isso, as empresas que têm múltiplas relações com seus parceiros tendem a obter ainda mais benefícios (BECKMAN; HAUNSCHIL, 2002).

Um elemento central para que ocorra a aprendizagem é o desenvolvimento e a manutenção de confiança entre os envolvidos em relações interorganizacionais (HIBBERT et al., 2010). A confiança constitui condição prévia para uma enriquecida, significativa e continuada troca de conhecimentos (MUTHUSAMY; WHITE, 2005). Nesse sentido confiança é a expectativa de que a outra parte se comportará de maneira justa e previsível, em particular quando o potencial para oportunismo está presente (ZAHEER; GÖZÜBÜYÜK; MILANOV, 1998). Por isso, não se trata de um conceito unidimensional (ROUSSAEU et al, 1998). A confiança é fator explicativo para a cooperação, facilitando as relações de trocas (WOOLTHUIS; HILLEBRAND; NOOTEBOOM, 2005; B ACHMANN; ZAHE$E R, 2008)$. Além disso o processo coletivo de aprendizagem é forta- 
lecido por relacionamentos de longo prazo baseados em confiança (LARSSON et al., 1998). À medida que a confiança é estabelecida cada organização fica mais disposta a aumentar o seu compromisso com a parceria, aprendendo a confiar e a aprender (LUBATKIN; FLORIN; LANE, 2001).

Diante de tais pressupostos básicos sobre o papel da confiança no processo de aprendizagem, a abordagem sociológica possibilita lançar luzes sobre algumas das dimensões negligenciadas nos estudos sobre aprendizado em diferentes níveis, como a aprendizagem da rede, justamente em razão das características das redes de empresas. Tais estruturas organizacionais dependem, em grande medida, da interação de seus participantes e da sua capacidade de confiar, compartilhar recursos, conhecimentos e experiências, gerando sinergias.

\subsection{Níveis de aprendizagem}

Além da discussão sobre as perspectivas teóricas sobre como ocorre o processo de aprendizagem os estudos analisam também os níveis em que sucede a aquisição de conhecimento, desde indivíduos e grupos que aprendem em um ambiente organizacional até o aprendizado no nível da organização, bem como nas organizações por meio dos interfaceadores. Em estudo recente Nogueira e Odelius (2015) confirmam que ainda existem muitos desafios para as pesquisas sobre $\mathrm{AO}$, dentre eles o referente aos níveis de aprendizagem, sendo que cada um exige um modelo de análise adequado.

Os acadêmicos que analisam indivíduos e grupos estão, na verdade, focando a aprendizagem individual e de grupo em um contexto organizacional (CROSSAN et al., 1995). Tal como apontam Silva, Vieira, Kubo e Bispo (2015), a aprendizagem organizacional é mais do que a soma da do processo de aquisição de conhecimento dos indivíduos e grupos que constituem a organização. Ocorrem mudanças nas propriedades da organização, como sistemas, estruturas, procedimentos e cultura, modificando padrões de ação como rotinas e estratégias (HUYSMAN, 1999). A confirmação da AO se dá pela manutenção de padrões comportamentais - mesmo que haja troca de pessoas na organização (KNIGHT, 2002).

Mais recentemente, o interesse acadêmico pela cooperação empresarial e pela formação de relações interorganizacionais estimulou 
o desenvolvimento de pesquisas sobre a AIO por meio de redes (INKPEN, 1996; LARSSON et al., 1998; CHILD, 2001; TEIXEIRA; GRZYBOVSKI; BEBER, 2009) e pela aprendizagem das próprias redes (KNIGHT, 2002; KNIGHT; PYE, 2005; WHITE, 2008). A aprendizagem interorganizacional (AIO) é referida pela literatura como aquela que ocorre no contexto de pares ou de grupos de organizações (BAPUJI; CROSSAN, 2004) que cooperam proativamente em diversos formatos de relações interorganizacionais (KNIGHT, 2002). O foco desses estudos geralmente é na apropriação de aprendizagem pela organização individual a partir da relação com outras organizações (LARSSON et al., 1998; CHILD, 2001; FLORÉN; TELL, 2004; ESTIVALETE; PEDROZO; BEGNIS, 2008), com o objetivo de influenciar positivamente o desempenho dos envolvidos (MOZZATO; BITENCOURT, 2014).

Nessa linha de pensamento, Crossan, Lane e White (1999) identificaram a aprendizagem em nível interorganizacional no processo contínuo de aprendizagem, mas não o incluíram no quadro conceitual analítico proposto por elas. Visando reforçar a importância desse quarto nível da aprendizagem e a necessidade de sua melhor compreensão, Mozzato e Bitencourt (2014) o incluem no modelo analítico de Crossan, Lane e White (1999). Assim, Mozzato, Bitencourt e Grzybovski (2015) legitimam a proposta de Crossan, Lane e White (1995), Knight (2002), Bapuji e Crossan (2004), Knight e Pye (2005) e Crossan, Maurer e White (2011). Elas também sugerem um construto com categorias analíticas para a melhor compreensão desse quarto nível da aprendizagem, com o intuito de contribuir para com o avanço dos estudos da mesma nas relações interorganizacionais. Mozzato e Bitencourt (2014) afirmam que é nesse quarto nível de aprendizagem que ocorre a cooperação interorganizacional. Entende-se, por conseguinte, que as estratégias relacionais estabelecidas nesse nível podem gerar a aprendizagem da rede, como denominam Knight (2002), Knight e Pye (2005) e White (2008).

Já na perspectiva de Knight (2002) há evidências de que a noção de aprendizagem pode ser aplicada em diferentes níveis, como grupos e organizações. Uma vez que as redes interorganizacionais representam o nível seguinte de organização, é lógico argumentar que as redes também podem aprender. A questão chave, portanto, não é 
se as redes podem aprender, mas como as elas aprendem (KNIGHT, 2002). O aprendizado da rede pode ser entendido como mais do que a soma da aprendizagem de indivíduos, grupos ou organizações que a compõem. White (2008) esclarece que a aprendizagem da rede é diferente de rede de aprendizado, pois esta última é vista como a interação entre um grupo de organizações com o propósito explícito de aprenderem juntas, entre elas, ou por meio de suas interações.

A aprendizagem da rede consiste em mudanças nos atributos dela própria, como processos de interação e estruturas. Trata-se de um grupo de organizações aprendendo sobre processos em rede para aumentar a sua efetividade da rede (WHITE, 2008). Evidências de estruturas cognitivas compartilhadas, como normas ou interpretações, assim como práticas coletivas estabelecidas em rede, servem de referência para confirmar a ocorrência de aprendizado da rede (KNIGHT, 2002). Isto é, aprendizagem da rede é a aprendizagem de um grupo de organizações enquanto grupo. Se por meio da interação ocorrem mudanças no comportamento ou na estrutura cognitiva do grupo, pode-se afirmar que o coletivo de organizações aprendeu e não somente as organizações individuais que fazem parte dele (KNIGHT, 2002; WHITE, 2008). Nesse caso, a rede aprendeu.

Quando não ocorrem mudanças em propriedades no nível da rede, não é possível afirmar que houve aprendizagem da rede. Se, por exemplo, ocorrem mudanças em uma ou mais organizações, mas estas não têm efeito em propriedades da rede, pode ter ocorrido $\mathrm{AIO}$, mas não aprendizagem da rede (KNIGHT; PYE, 2005). Esta última caracteriza-se por mudanças nas práticas da rede, interpretações da rede e estruturas da rede, em três níveis que vão do mais simples ao mais complexo. Portanto, na medida em que ocorre aprendizagem da rede o grupo de empresários aprende sobre os processos colaborativos e compartilha interpretações comuns sobre o que é a rede e como ocorrem as relações interpessoais. As mudanças precisam ser difundidas e duradouras para que sejam consideradas resultados da aprendizagem da rede, mas não precisam necessariamente serem universais ou uniformes na rede como um todo (KNIGHT; PYE, 2005).

Baseadas em um estudo longitudinal de caráter qualitativo com uma rede de organizações, Knight e Pye (2005) analisaram a 
aprendizagem do coletivo utilizando o modelo contexto-conteúdo-processo (CCP) de Pettigrew (1987). Tal modelo consiste em descrever os antecedentes imediatos e os mais distantes de um processo de mudança, em uma perspectiva holística, utilizando uma abordagem processual e contextual. O contexto externo refere-se ao ambiente social, econômico e político em que a empresa (neste caso, a rede) opera. Já o contexto interno consiste na estrutura, cultura e contexto político interno em que a mudança será analisada. O conteúdo refere-se às mudanças em si, ao passo que o processo analisa as ações, reações e interações das várias partes envolvidas na mudança. Conforme defende Pettigrew (1987), o "porquê" da mudança deriva da análise do contexto interno e externo, ao passo que o "o quê" mudou e é analisado no conteúdo da mudança. Por sua vez, o "como" pode ser identificado a partir da análise do processo. A proposta de Knight e Pye (2005) para analisar a aprendizagem da rede, usando como referência o modelo contexto-conteúdo-processo de Pettigrew (1987), é sumarizada na Figura 2.

\section{Figura 2 - Modelo de aprendizagem da rede}

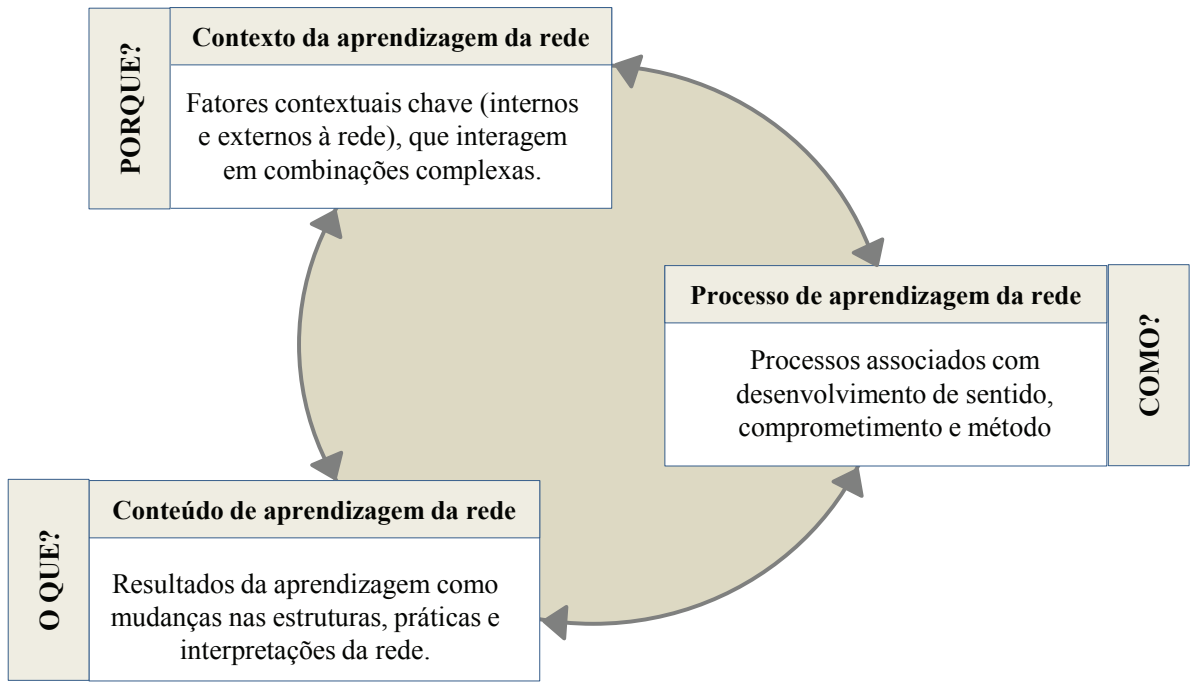

Fonte: Knight e Pye (2005, p. 383). 
Analisando uma rede de prestadores de serviços de atenção à saúde no Reino Unido, Knight e Pye (2005) fizeram uma rica descrição do contexto interno e externo de atuação da rede e identificaram o conteúdo de mudanças nas práticas, na estrutura e nas interpretações do grupo. Essas mudanças caracterizaram episódios de aprendizagem da rede. Seguindo o modelo CCP de Pettigrew (1987), as autoras estudaram os processos subjacentes às mudanças identificadas, isto é, as ações e interações por meio das quais mudanças no nível da rede ocorreram. Tais processos consistem em desenvolver significados entre os atores, em relação a um determinado tema, desenvolver comprometimento entre os atores para realizar algum tipo de mudança e desenvolver método, no sentido de definir e organizar tecnologias, sistemas, rotinas e recursos. Embora se pudesse imaginar que esse processo de aprendizagem ocorre por meio de etapas sequenciais de desenvolver significados, comprometimento e método, o estudo empírico revelou que essas três ações podem ocorrer em qualquer ordem e mesmo simultaneamente.

Knight e Pye (2005) buscaram identificar episódios de aprendizagem da rede, na medida em que o estabelecimento de relacionamentos entre indivíduos é propício para tal ocorrência. Por episódios de aprendizagem da rede, com base em Knight (2002) e Knight e Pye (2005), entende-se o fluxo das atividades cotidianas que se dão tanto em espaços formais como informais. Dito de outra maneira, trata-se de ações e interações que ocorrem entre os diferentes indivíduos que propiciam acontecimentos e experiências de aprendizagem (eventos de aprendizagem), desencadeando os episódios de aprendizagem, os quais têm impacto maior ou menor, mais direto ou indireto para os envolvidos. Tais episódios podem ser analisados em seu conteúdo, visando ao que foi aprendido (KNIGHT; PYE, 2005), como pode direcionar o foco de investigação para a verificação da ocorrência desses episódios, analisando-se a importância deles para os envolvidos.

Como se vê, a intercooperação de redes empresariais exige a interação, a confiança e a cooperação interorganizacional em espaços formais ou informais que possibilitam o desencadeamento dos episódios de aprendizagem. O processo de aprendizagem facilita a difusão do conhecimento e melhora o desempenho das organi- 
zações envolvidas (EBERS; JARILLO, 1997; MUTHUSAMY; WHITE, 2005; VERSCHOORE; B ALESTRIN, 2008; ZAHEER; GÖZÜBÜYÜK; MILANOV, 2010; MOZZATO; BITENCOURT; GRZYBOVSKI, 2015).

A abordagem teórica da AIO sob a perspectiva sociológica também pode servir como base ontológica e epistemológica alternativa para contribuir para o avanço dos estudos sobre a aprendizagem das redes, visto que mesmo que ainda incipiente nos estudos brasileiros já serve como base para os estudos sobre AO. Justamente em razão da carência de estudos empíricos sobre o processo da aprendizagem sob a perspectiva sociológica e, sobretudo, pelo fato de que o processo de intercooperação e fusão de redes empresariais é assunto emergente, apresenta-se na sequência a proposição de esquema conceitual analítico para sua melhor compreensão.

\section{INTERCOOPERAÇÃO E FUSÃO DE REDES EMPRESARIAIS: PROPOSIÇÃO DE UM FRAMEWORK DE ANÁLISE}

Para a proposição do framework do processo de intercooperação e fusão de redes torna-se necessária a análise dos episódios de aprendizagem que ocorrem nas fronteiras organizacionais por meio das interações colaborativas tanto para a implementação, a condução como para a consolidação do processo. Interações colaborativas em diferentes espaços sociais geram episódios de aprendizagem em todos os níveis (individual, grupal, organizacional e interorganizacional), possibilitando a aprendizagem da rede ao se considerar o contexto, o processo e o conteúdo.

A aprendizagem não ocorre de forma independente do contexto, sendo influenciada pelo ambiente social, econômico ou político em que a rede atua ou ainda de seu próprio ambiente interno (PETTIGREW, 1987; SANDBERG; TSOUKAS, 2011). Mudanças nesses ambientes (interno e externo) podem ser catalisadoras de novas estratégias. A entrada de um novo concorrente no mercado de atuação das empresas da rede, por exemplo, pode impactar negativamente as empresas e minimizar os benefícios gerados pela cooperação. Ganhos gerados pela ação conjunta, que antes refletiam em benefícios significativos para os participantes, podem ser afetados pela dinâmica do mercado e da concorrência. 
No ambiente interno da rede podem surgir discussões sobre o aumento da competição, questões relacionadas à confiança interpessoal e às dificuldades enfrentadas pela rede. $O$ contexto social influencia a ocorrência da aprendizagem (SANDBERG; TSOUKAS, 2011), assim como a questão do tempo salientada por Berends e Antonacopoulou (2014).

Todos esses acontecimentos são trazidos pelos empresários para os espaços de interação da rede (reuniões, assembleias, grupos de trabalho), gerando episódios de aprendizagem que, por sua vez, podem levar à aprendizagem da rede sobre a adoção de novas estratégias, como sintetizado na primeira premissa desse estudo: fatores contextuais internos e externos estimulam a aprendizagem da rede quanto à necessidade de adotar estratégias de intercooperação e fusão.

Sendo a aprendizagem uma atividade inerentemente relacional, decorrente das interações cotidianas (GHERARDI; NICOLINI; ODELLA, 1998; LAVE; WENGER, 1991; GHERARDI; NICOLINI, 2001; NICOLINI; GHERARDI; YANOW, 2003; MASON; LEEK, 2008; CORRADI; GHERARDI; VERZELLONI, 2010), ela é o resultado desses episódios de aprendizado (KNIGHT, 2002; KNIGHT; PYE, 2005) que dá sentido às estratégias adotadas na intercooperação e fusão de redes empresariais.

À medida que as discussões geradas no ambiente da rede evoluem e se consolidam ocorre um desenvolvimento gradual de sentido entre os empresários no que se refere à necessidade e à viabilidade de cooperar com outras redes. Sucessivos episódios de aprendizagem reforçados por novas mudanças no contexto interno e externo da rede estimulam um número maior de empresários a se comprometer com a estratégia de intercooperação e fusão. Quando o desenvolvimento de sentido e comprometimento se torna mais abrangente no grupo iniciam-se novos episódios de aprendizagem para discussão e definição de métodos que permitam a efetiva realização das estratégias. Essas ideias são sintetizadas na segunda premissa: o desenvolvimento de sentido, comprometimento e método direciona a aprendizagem da rede para a execução de estratégias de intercooperação e fusão.

Como resultado da dinâmica interorganizacional - que envolve a transmissão, a troca de conhecimentos (VAN WIJK; JANSEN; 
LYLES, 2008) e fundamentalmente o aprendizado da rede - geralmente ocorrem mudanças que constituem o próprio conteúdo desse aprendizado (PETTIGREW, 1987, KNIGHT; PYE, 2005). Igualmente advêm alterações nas práticas, estruturas e interpretações da rede. Nessa etapa do processo a compreensão da necessidade de estabelecer uma estratégia de intercooperação e fusão possivelmente já está sedimentada na rede. É normal sucederem mudanças em práticas internas como, por exemplo, a designação de um grupo de empresários que será responsável pelo contato e negociação com outras redes para dar início à estratégia. Pode também continuar a ocorrer episódios de aprendizagem à medida que os empresários discutem a adoção da estratégia e dão início a ela, como sintetizado na terceira premissa: mudanças nas estruturas, práticas e interpretações consolidam a aprendizagem da rede no processo de intercooperação e fusão.

Deve-se destacar que, apesar da apresentação sequencial, as etapas do modelo contexto-conteúdo-processo (PETTIGREW, 1987; KNIGHT; PYE, 2005) podem ocorrer em sobreposição e inter-relação. Ao mesmo tempo em que ocorrem episódios de aprendizagem que estimulam a aprendizagem da rede, têm lugar alterações no ambiente externo e interno que podem afetar essa aprendizagem. O conteúdo da aprendizagem da rede da mesma maneira se modifica com as mudanças de contexto e processo, de forma recursiva. Portanto, como se vê, trata-se de elementos que interagem dinamicamente, não sendo possível analisá-los de maneira linear na tentativa de apreender a aprendizagem da rede (Está correto assim).

Além disso, a confiança atua como facilitadora em todas as etapas da aprendizagem da rede, potencializando e acelerando o intercâmbio de conhecimentos. A literatura organizacional reconhece que a confiança entre os membros de uma relação interorganizacional funciona como propulsor da cooperação (WOOLTHUIS; HILLEBRAND; NOOTEBOOM, 2005; BACHMANN; ZAHEER, 2008) e favorece trocas de informação e conhecimento (WEGNER; ANTONELLO, 2012). Quando existe alto nível de confiança e ela está amplamente distribuída no grupo, acontecimentos relevantes do contexto interno e externo da rede são compartilhados mais rapidamente e estimulam o aprendizado da rede. O desenvolvimento 
de sentido, comprometimento e método da mesma forma é facilitado quando existe alta densidade de relações de confiança entre os membros, já que há menor receio de ações oportunistas (WEGNER; MISOCSKY, 2010). Por conseguinte, se constata que a confiança favorece mudanças nas estruturas, práticas e interpretações que consolidam a aprendizagem da rede. Ao inverso, um baixo nível de confiança possivelmente impede a aprendizagem da rede ou a torna mais lenta. Esses argumentos são sintetizados na quarta premissa: o nível de confiança entre as organizações de uma rede interfere no processo de aprendizagem da rede.

Esquematizadas as premissas deste estudo, na sequência delineiam-se proposições que sustentam o framework proposto para a análise da intercooperação e fusão de redes empresariais sob a perspectiva sociológica da aprendizagem da rede. No primeiro momento a aprendizagem da rede funciona como um catalisador da decisão de adotar uma estratégia de intercooperação, conforme esquematizado a seguir:

Proposição 1: Episódios de aprendizagem relacionados ao contexto, conteúdo e processo interferem na decisão da rede pela estratégia de intercooperação.

A adoção da estratégia de intercooperação coloca a rede em contato com outras redes, e ações conjuntas passam a ser realizadas pelos participantes dos grupos envolvidas. Nessa etapa a estratégia de intercooperação gera novos episódios de aprendizagem, como apresentado na Proposição 2:

Proposição 2: A implementação da estratégia de intercooperação leva a novos episódios de aprendizagem relacionados ao contexto, conteúdo e processo.

Finalmente, caso a estratégia de intercooperação seja bemsucedida e haja resultados positivos para os participantes, novos episódios de aprendizagem podem levar à opção pela fusão das redes, conforme a Proposição 3: 
Proposição 3: Episódios de aprendizagem relacionados ao contexto, conteúdo e processo na estratégia de intercooperação interferem na decisão pela fusão das redes.

Com base nas proposições, apresenta-se o referido framework na Figura 3.

\section{Figura 3 - Framework da aprendizagem da rede na intercooperação} e fusão de redes de empresas

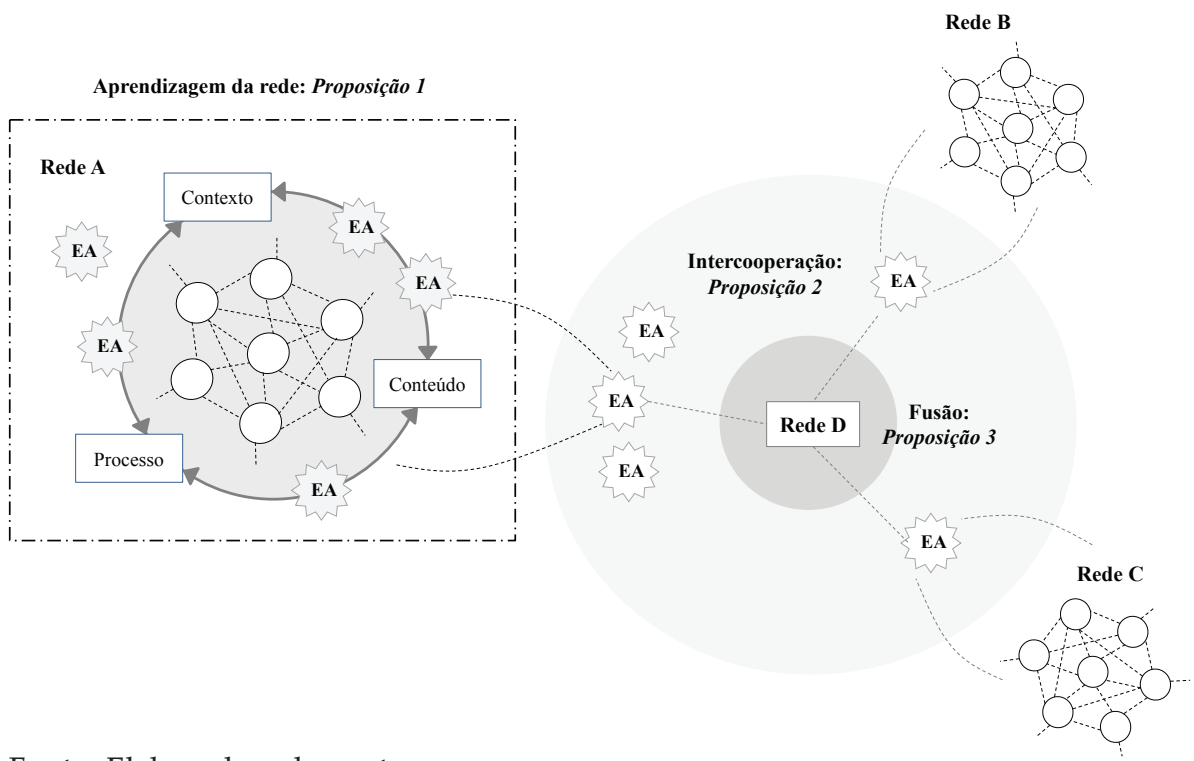

Fonte: Elaborada pelos autores.

Para que a intercooperação seja iniciada é necessário que ocorra aprendizagem em cada uma das redes participantes do processo. Na Figura 3 destaca-se que episódios de aprendizagem (EA) ocorreram na Rede A, mas também na Rede B e na Rede C, configurando o aprendizado da rede.

Refletindo as proposições do framework, a Figura 3 ilustra que ocorrem EA estimulados pelo contexto em que a rede está operando, o que caba por gerar novos episódios no processo de aprendizagem do grupo, influenciando o conteúdo dessa aprendizagem. Tudo isso se dá em uma sequência não linear que leva essa rede a optar 
pela estratégia de intercooperação com outras (Proposição 1). Esse mesmo ciclo de aprendizagem ocorre igualmente nas outras redes, ainda que com características distintas e por meio de eventos de aprendizagem diferentes. Ocorrendo a opção pela intercooperação, essa impele a novos episódios de aprendizagem que também se dão no contexto, conteúdo e processo (Proposição 2). Por fim, os episódios de aprendizagem que continuam ocorrendo nesses níveis de contexto, conteúdo e processo na intercooperação interferem na opção pela fusão das redes (Proposição 3). Logo, é a partir da aprendizagem da rede que estas optam pela intercooperação, podendo chegar até à fusão. Ainda, essa mesma aprendizagem da rede se encontra presente e é necessária para a condução e consolidação da estratégia de intercooperação e fusão.

\section{CONSIDERAÇões FINAIS}

Partindo da perspectiva sociológica da aprendizagem organizacional, este ensaio teve por objetivo central propor um framework para aprofundar o entendimento sobre como o processo de aprendizagem das redes interorganizacionais pode conduzir a estratégias de intercooperação e fusão. Com base na argumentação apresentada, foram elaboradas três proposições que unem os conceitos fundamentais dos respectivos temas.

Como principal contribuição, o ensaio propõe que as transformações nas redes de empresas são resultado de um processo de aprendizado que transcende as organizações individuais, configurando aprendizado da rede. Do ponto de vista gerencial isso implica em que gestores de redes devem promover ativamente a interação dos participantes e estar atentos aos episódios de aprendizagem que produzem efeitos sobre a rede. Tais ocorrências, que se dão no dia a dia por meio das diferentes interações, constituem a base que poderá se transformar em estratégias efetivas que levem à evolução e consolidação das redes. Mas é importante enfatizar que a definição sobre a estratégia de intercooperação e fusão dependerá do resultado do aprendizado das redes.

Pela perspectiva sociológica este processo de aprendizado se dá por meio das interações que ocorrem dentro e entre as redes e é destacado em eventos de aprendizagem resultantes de situações 
contextuais impostas tanto por seu ambiente externo (fora das redes) como interno (dentro das redes). Uma vez que há aprendizado, este se verifica na forma de mudança na gestão ou estrutura da rede. No framework proposto esta mudança se refere ao conteúdo do aprendizado, enquanto o processo é determinado pelas interações, as quais são condicionadas por variáveis ambientais que configuram o contexto. Pesquisas empíricas com redes empresariais que tenham realizado estratégias de intercooperação e fusão podem contribuir para a validação e aperfeiçoamento do framework proposto. Além disso, tais estudos ainda contribuem para as escassas investigações empíricas sobre aprendizagem no nível da rede, proposta por Knight (2002), Knight e Pye (2005).

O estudo apresentado colabora, da mesma forma, para o avanço das pesquisas sobre intercooperação e fusão de redes empresariais, raramente abordadas na literatura. Pesquisas podem avançar na investigação sobre fatores de estímulo às interações que promovem o aprendizado do coletivo, assim como identificar as variáveis ambientais que facilitam ou direcionam o processo de aprendizado da rede para que ocorra a intercooperação e fusão.

\section{REFERÊNCIAS}

ANTONACOPOULOU, E. P. The relationship between individual and organizational learning: New evidence from managerial learning practices. Management Learning, v.37, n.4, p.455-473. Sage Publishing, 2006.

ANTONACOPOULOU, E.; CHIVA, R. The social complexity of organizational learning: the dynamics of learning and organizing. Management Learning, v.38, n.3, p.277-295 Sage Publishing, 2007.

BACHMANN, R.; ZAHEER, A. Trust in interorganizational relations. In: GOTCHEVA, N.; CROPPER, S.; EBERS, M.; HUXHAM, C.; RING, P. S. The Oxford handbook of inter-organizational relations. Oxford: Oxford University Press. p. 533-554, 2008.

BALESTRIN, A.; VERSCHOORE, J. Aprendizagem e inovação no contexto das redes de cooperação entre pequenas e médias empresas. Organizações \& Sociedade, v.17, n.53, p.311330. Salvador, Programa de Pós-Graduação em Administração da Universidade Federal da Bahia - UFBA, 2010.

BAPUJI, H.; CROSSAN, M. From questions to answers: reviewing organizational learning research. Management Learning, v.35, n.4, p.397-417. Sage Publishing, 2004.

BECKMAN, C. M.; HAUNSCHILD, P. R. Network learning: The effects of partners' heterogeneity of experience on corporate acquisitions. Administrative Science Quarterly, v.47, n.1, p.92-124. Ithaka, Samuel Curtis Johnson Graduate School of Management at Cornell University, 2002. 
BERENDS, H.; ANTONACOPOULOU, E. Time and Organizational Learning: A Review and Agenda for Future Research. International Journal of Management Reviews, v.16, n.4, p.437453. John Wiley \& Sons Ltd and the British Academy of Management, 2014.

BOERNER, C.; MACHER, J.; TEECE, D. A review and assessment of organizational learning in economic theories. In: DIERKES, M.; ANTAL, A. B.; CHILD, J.; NONAKA, I. (Org.). The handbook of organizational learning and knowledge. Oxford: Oxford University Press, 2001, p. 89-117.

BRASS, D. J.; GALASKIEWICZ, J.; GREVE, H. R.; TSAI, W. Taking stock of networks and organizations: A multilevel perspective. Academy of Management Journal, v.47, n.6, p.795817. John Wiley \& Sons Ltd and Society for the Advancement of Management Studies, 2004.

CHILD, J. Learning through strategic alliances. In: DIERKES, M.; ANTAL, A. B.; CHILD, J.; NONAKA, I. (Eds.), Organizational Learning and knowledge, p. 657-680, Oxford: Oxford University Press, 2001.

CHIVA, R.; HABIB, J. A framework for organizational learning types: generative, adaptive and zero learning. Journal of Management and Organization, v. 21, n. 3, p. 350-368. Australian and New Zealand Academy of Management, 2015.

CORRADI, G.; GHERARDI, S.; VERZELLONI, L. Through the practice lens: where is the bandwagon of practice-based studies heading? Management Learning, v.41, n.3, p.265-283. Sage Publishing, 2010.

CORSARO, D.; CANTÙ, C.; TUNISINI, A. Actors' heterogeneity in innovation networks. Industrial Marketing Management, v.41, n.5, p.780-789. Elsevier, 2012.

CROSSAN, M. M.; LANE, H. W.; WHITE, R.; DJURFELDT, L. Organizational learning: Dimensions for a theory. The International Journal of Organizational Analysis, v.3, n.4, p.337-360. Emerald Publishing, 1995.

CROSSAN, M. M.; LANE, H. W.; WHITE, R. E. An organizational learning framework: From intuition to institution. Academy of Management Review, v.24, n.3, p.522-537. Academy of Management, 1999.

CROSSAN, M. M.; MAURER, C. C.; WHITE, Roderick E. Reflections on the 2009 AMR decade award: do we have a theory of organizational learning? Academy of Management Review, v.36, n.3, p.446-460. Academy of Management, 2011.

CZARNIAWSKA, B. Anthropology and Organizational Learning. In: DIERKES, M.; ANTAL A. B.; CHILD, J.; NONAKA, I. Handbook of Organizational Learning and Knowledge, p.118-136. Oxford: Oxford University Press, 2001.

EASTERBY-SMITH, M. Disciplines of organizational learning: contributions and critiques. Human Relations, v.50, n. 9, p.1085-1113. Sage Publishing, 1997.

EBERS, M.; GRANDORI, A. The Forms, Costs, and Development Dynamics of Inter-Organizational Networking. In: EBERS, M. The formation of inter-organizational networks. New York: Oxford University Press, 1999.

EBERS, M.; JARILLO, J. C. The construction, forms, and consequences of industry networks. International Studies of Management \& Organization, v.27, n.4, p.3-21. Taylor \& Francis, 1997.

ESTIVALETE, V.; PEDROZO, E.; BEGNIS, H. S. M. Em busca da ação coletiva: estratégias de aprendizagem interorganizacional adotadas pelas organizações que estabelecem relacionamentos horizontais em redes. Base, v.5, n.3, 224-235. São Leopoldo, Editora Unisinos, 2008. 
FLORÉN, H.; TELL, J. The emergent prerequisites of managerial learning in small firm networks. Leadership \& Organization Development Journal, v.25, n.3, p.292-307. Emerald, 2004. GHERARDI, S. Introduction: the critical power of the "practice lens". Management Learning, v. 40, n. 2, p. 115-128. Sage Publishing, 2009a.

GHERARDI, S. Practice? It's a matter of taste! Management Learning, v. 40, n. 5, p. 535-550. Sage Publishing, 2009b.

GHERARDI, S. To start practice theorizing anew: The contribution of the concepts of agencement and formativeness. Organization, v.23, n.5, p.680-698. Sage, 2015.

GHERARDI, S. How to conduct a practice-based study: Problems and methods. Cheltenham: Edward Elgar Publishing, 2012.

GHERARDI, S. Organizational Knowledge: The Texture of Workplace Learning. Oxford: Blackwell, 2006.

GHERARDI, S.; NICOLINI, D. The sociological foundations of organizational learning. In: DIERKES, M.; ANTAL A. B.; CHILD, J.; NONAKA, I. Handbook of Organizational Learning and Knowledge, p. 35-60. Oxford: Oxford University Press, 2001.

GHERARDI, S.; NICOLINI, D.; ODELLA, F. Toward a social understanding of how people learn in organizations the notion of situated curriculum. Management Learning, v. 29, n. 3, p. 273-297. Sage Publishing, 1998.

GHERARDI, S. How the turn to practice may contribute to working life studies. Nordic Journal of working life studies, v. 5, p. 13-25. Aalborg University, 2015.

GOMES-CASSERES, B. Group vs. group: how alliance networks compete. Harvard Business Review, v. 72, n. 4, p. 62-67. Harvard, 1994.

GRONUM, S.; VERREYNNE, M.; KASTELLE, Tim. The Role of Networks in Small and Medium-Sized Enterprise Innovation and Firm Performance. Journal of Small Business Management, v. 50, n. 2, p. 257-282. International Council for Small Business, 2012.

GRO $\beta, W$. Strategische Entwicklungsrichtungen für erbundgruppen des Handels. In: WIESELHUBER (Ed.). Erfolg im Handel: Praxis des Kooperationsmanagements. Frankfurt am Main: Deutscher Fachverlag, 2003.

GULATI, R. Alliances and networks. Strategic Management Journal, v. 19, n. 4, p. 293-317, 1998.

HIBBERT, P.; HUXHAM, C.; SYDOW, J.; LERCH, F. Barriers to process learning: Authority and anomie in regional clusters. Management Learning, v. 41, n. 4, p. 453-471. Sage Publishing, 2010.

HUIZINGH, E. KRE. Open innovation: State of the art and future perspectives. Technovation, v. 31, n. 1, p. 2-9. Elsevier, 2011.

HUYSMAN, M. Balancing biases: a critical review of the literature on organizational learning. Organizational Learning and the Learning Organization, p. 59-74. Lancaster, Sage, 1999.

INKPEN, A. C. Creating knowledge through collaboration. California Management Review, v. 39, n. 1, p. 123-140. Berkeley, Sage, 1996.

JANOWICZ-PANJAITAN, M.; NOORDERHAVEN, N. G. Trust, calculation, and interorganizational learning of tacit knowledge: An organizational roles perspective. Organization Studies, v. 30, n. 10, p. 1021-1044. Sage Publishing, 2009. 
KILDUFF, M.; TSAI, W. Social networks and organizations. London: Sage, 2003.

KNIGHT, L. Network learning: Exploring learning by interorganizational networks. Human Relations, v. 55, n. 4, p. 427-454. Sage Publishing, 2002.

KNIGHT, L.; PYE, A. Network learning: An empirically derived model of learning by groups of organizations. Human Relations, v. 58, n. 3, p. 369-392. Sage Publishing, 2005.

LAMBERT, D. M.; COOPER, M. C.; PAGH, J. D. Supply chain management: implementation issues and research opportunities. The International Journal of Logistics Management, v. 9, n. 2, p. 1-20. Emerald, 1998.

LARSSON, R.; BENGTSSON, L.; HENRIKSSON, K.; SPARKS, J. The interorganizational learning dilemma: Collective knowledge development in strategic alliances. Organization Science, v. 9, n. 3, p. 285-305. Catonsville, The Institute for Operations Research and the Management Sciences, 1998.

LAVE, J.; WENGER, E. Situated learning: Legitimate peripheral participation. Cambridge: Cambridge University Press, 1991.

LAZZARINI, S. G. The impact of membership in competing alliance constellations: Evidence on the operational performance of global airlines. Strategic Management Journal, v. 28, n. 4, p. 345-367. Academy of Management, 2007.

LICHTENTHALER, U. Open innovation: Past research, current debates, and future directions. The Academy of Management Perspectives, v. 25, n. 1, p. 75-93. Academy of Management, 2011.

LUBATKIN, M.; FLORIN, J.; LANE, P. Learning together and apart: A model of reciprocal interfirm learning. Human Relations, v. 54, n. 10, p. 1353-1382. Sage Publishing, 2001.

MACDONALD, P.; CROSSAN, M. Learning to innovate: the process of learning between diverse organizations. In: Proceedings of Organisational Learning, Knowledge and Capabilities Conference 2010.Boston (USA), Northeastern University, 2010.

MAIER, G.; W.; PRANGE, C.; ROSENSTIEL, L. Psychological perspectives of organizational learning. In: DIERKIS, M.; ANTAL; A. B.; CHILD, J.; NONAKA, I. Handbook of organizational learning and knowledge, p. 14-34. Oxford: Oxford University Press, 2001.

MASON, K. J.; LEEK, S. Learning to build a supply network: an exploration of dynamic business models. Journal of Management Studies, v. 45, n. 4, p. 774-799. John Wiley \& Sons Ltd and Society for the Advancement of Management Studies, 2008.

MOZZATO, A. R.; BITENCOURT, C. C.; GRZYBOVSKI, D. The Interorganizational Level in the Learning Continuum: Analytic Conceptual Scheme. International Business Research, v. 8, n. 4, p. 94. Canadian Center of Science and Education, 2015.

MOZZATO, A. R.; BITENCOURT, C. C. Understanding interorganizational learning based on social spaces and learning episodes. BAR-Brazilian Administration Review, v. 11, n. 3, p. 284-301. ANPAD - The Brazilian Academy of Management, 2014.

MUTHUSAMY, S. K.; WHITE, M. A. Learning and knowledge transfer in strategic alliances: a social exchange view. Organization Studies, v. 26, n. 3, p. 415-441. Sage, 2005.

NICOLINI, D.; GHERARDI, S.; YANOW, D. Knowing in organizations: A practice-based approach. London: M.E. Sharpe, 2003.

NOGUEIRA, R. A.; ODELIUS, C. C. Desafios da Pesquisa em Aprendizagem Organizacional. Cadernos EBAPE.BR, v. 13, n. 1, p.83-102. Rio de Janeiro, Fundação Getúlio Vargas, 2015. 
PETTIGREW, A. M. Context and action in the transformation of the firm. Journal of Management Studies, v. 24, n. 6, p. 649-670. John Wiley \& Sons Ltd and Society for the Advancement of Management Studies, 1987.

SEBRAE, Serviço Brasileiro de Apoio às Micro e Pequenas Empresas. Mapeamento das centrais e redes de negócio - 2011. Brasília: SEBRAE, 2012.

ROSSI, A. do C. S. Cooperativismo à luz dos princípios constitucionais. Curitiba: Jurua, 2005.

ROUSSEAU, D. M.; SITKIN, S. B.; BURT, R. S.; CAMERER, C. Not so different after all: A cross-discipline view of trust. Academy of Management Review, v.23, n. 3, 393-404. Academy of Management, 1998.

SANDBERG, J.; TSOUKAS, H. Grasping the logic of practice: Theorizing through practical rationality. Academy of Management Review, v. 36, n. 2, p. 338-360. Academy of Management, 2011.

SCHULZE, A.; BROJERDI, G.; KROGH, G. Those who know, do. Those who understand, teach. Disseminative capability and knowledge transfer in the automotive industry. Journal of Product Innovation Management, v. 31, n. 1, p. 79-97. Product Development and Management Association, 2014.

SILVA, E. C. A.; VIEIRA, A. M.; KUBO, E. K. M.; BISPO, M. S. Aprendizagem Organizacional no Setor Farmacêutico: uma Análise Multinível a Partir da Percepção dos Gestores. RACE: Revista de Administração, Contabilidade e Economia, v. 14, n. 3, p. 1091-1118, 2015.

SOARES, W. B.; WEGNER, D.; DOLCI, P. C. Fusão Como Estratégia de Crescimento de Redes de Cooperação Empresarial: Um Estudo de Caso em uma Rede de Farmácias. Iberoamerican Journal of Strategic Management (IJSM), v. 15, n. 1, p. 83-96. São Paulo, Programa de PósGraduação em Administração da Universidade Nove de Julho - UNINOVE, 2016.

STYHRE, A.; JOSEPHSON, P.; KNAUSEDER, I. Organization Learning in Non-writing Communities The Case of Construction Workers. Management Learning, v. 37, n. 1, p. 83-100. Sage Publishing, 2006.

TEIXEIRA, E. B.; GRZYBOVSKI, D.; BEBER, M. C. Cooperação e Aprendizagem Organizacional em Organizações Sociais Estruturadas em Redes de Cooperação: o Caso da Rede Integração Social. Redes, v. 14, n. 2, p. 134-157. Santa Cruz do Sul, Edunisc, 2009.

TODEVA, E. Business networks: strategy and structure. London: Routledge, 2006.

TOIGO, T.; ALBA, G. R. Programa Redes de Cooperação do estado do Rio Grande do Sul: Perfil das Redes de Empresas acompanhadas pela Universidade de Caxias do Sul. XIII Semead-Seminários em Administração. Anais... São Paulo: Edusp, 2010.

VAN WIJK, R.; JANSEN, J. J. P.; LYLES, M. A. Inter-and intra-organizational knowledge transfer: a meta-analytic review and assessment of its antecedents and consequences. Journal of Management Studies, v. 45, n. 4, p. 830-853. John Wiley \& Sons Ltd and Society for the Advancement of Management Studies, 2008.

VERSCHOORE, J. R.; BALESTRIN, A. Fatores relevantes para o estabelecimento de redes de cooperaçăo entre empresas do Rio Grande do Sul. RAC, v. 12, n. 4, p. 1043-1069. ANPAD The Brazilian Academy of Management, 2008.

WEGNER, D.; ALIEVI, R. M.; BEGNIS, H. S. M. The life cycle of small-firm networks: an evaluation of Brazilian business networks. BAR-Brazilian Administration Review, v. 12, n. 1, p. 39-62. ANPAD - The Brazilian Academy of Management, 2015. 
WEGNER, D.; ANTONELLO, C. S. Inter-organizational learning: a study of small-firm networks in Southern Brazil. African Journal of Business Management, v. 6, n. 1, p. 129-139. Academic Journals, 2012.

WEGNER, D.; MISOCSKY, M. C. Performance evaluation of small-firm networks: contributions of the interpretative approach. Organizações \& Sociedade, v. 17, n. 53, p. 345-361. Salvador, Programa de Pós-Graduação em Administração da Universidade Federal da Bahia - UFBA, 2010.

WEGNER, D.; PADULA, A. D. A influência de fatores contextuais na governança de redes interorganizacionais (RIOS). Gestão \& Planejamento, v. 14, n. 1, p. 116-136. Salvador, Programa de Pós-Graduação em Administração da Universidade Salvador - UNIFACS, 2012.

WEGNER, D.; PADULA, A. D. Estratégias de crescimento e a governança de redes horizontais de empresas: o caso da maior rede cooperativa de varejo de alimentos na Alemanha. Revista de Ciências da Administração, v. 13, n. 30, p. 220-248. Florianópolis, Editora da UFSC, 2011. WEGNER, D.; PADULA, A. D. Governance and management of horizontal business networks: an analysis of retail networks in Germany. International Journal of Business and Management, v. 5, n. 12, p. 74-88. Canadian Center of Science and Education, 2010.

WELLENBECK, M. T. Zentralregulierung der Nord-West-Ring-Grupppe. Zentes, Joachim/ Bernhard Swoboda (Hg.).: Perspektiven der Zentralregulierung. p. 248-273. Frankfurt/Main, Deutscher Fachverlag, 2001.

WHITE, L. Connecting organizations: Developing the idea of network learning in inter-organizational settings. Systems Research and Behavioral Science, v.25, n.6, p.701-716. John Wiley \& Sons Ltd , 2008.

WOOLTHUIS, R. K.; HILLEBRAND, B.; NOOTEBOOM, B. Trust, contract and relationship development. Organization Studies, v.26, n.6, p. 813-840. Sage Journals, 2005.

YSA, T.; SIERRA, V.; ESTEVE, M. Determinants of Network Outcomes: The Impact of Management Strategies. Public Administration, v.92, n. 3, p. 636-655. John Wiley \& Sons Ltd., 2014.

ZAHEER, A.; GÖZÜBÜYÜK, R.; MILANOV, H. It's the connections: The network perspective in interorganizational research. The Academy of Management Perspectives, v.24, n.1, p. 6277. Academy of Management, 2010.

Recebido em: 23-10-2017

Aprovado em: 21-11-2018

Avaliado pelo sistema double blind review.

Editor: Coordenação do PPGA/UMESP

Disponível em http://mjs.metodista.br/index.php/roc 